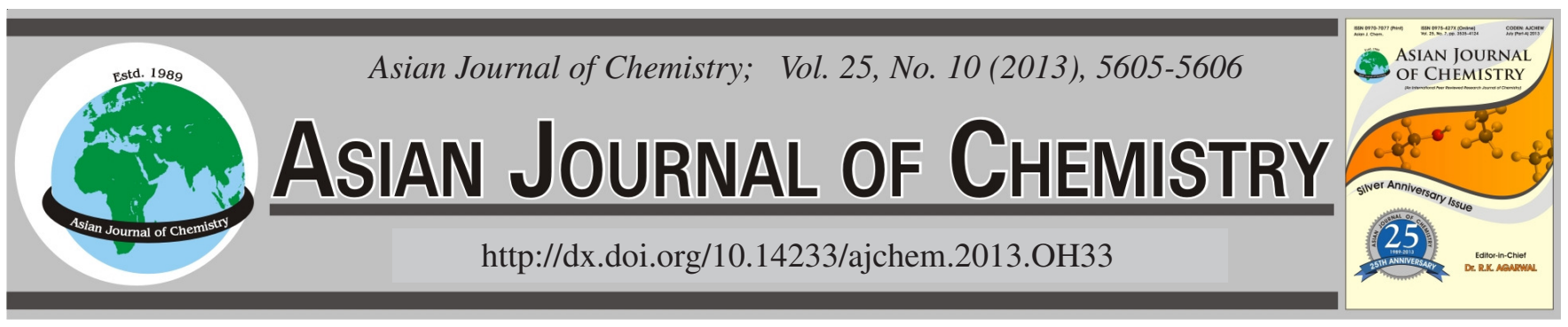

\title{
Ion-Exchange Processes in the Channels of Zeolites $\dagger$
}

\author{
T. MarsagishVILI ${ }^{1, *}$, M. MachaVARIANI $^{1}$, G. TatishVILI ${ }^{1}$, R. KhositashVILI $^{1}$ and N. LeKIShVILI ${ }^{2}$
}

${ }^{1}$ R. Agladze Institute of Inorganic Chemistry and Electrochemistry, Ivane Javakhishvili Tbilisi State University, Tbilisi, Georgia ${ }^{2}$ Ivane Javakhishvili Tbilisi State University, 1, Ilia Chavchavadze Ave., 0128 Tbilisi, Georgia

*Corresponding author: Email: tamaz.marsagishvili@gmail.com

Processes of non-adiabatic ion-exchange in the channels of zeolites have been considered. Calculations of rate constant of ion-exchange process in in the channels of zeolites, where one adsorbed particle is substituted for another, have been carried out. Analytical expressions for characteristic parameters of the process have been obtained.

Key Words: Zeolite, Ion-exchange, Non-adiabatic, Nanopores, Rate constant.

\section{INTRODUCTION}

Natural and synthetic zeolites have wide application in the gas separation installations, gas generators, purification plants, for creation of ionistors, super-condensers, accumulators. Their wide application is connected with specific structure of zeolites, form of their nanopores provide unique sorption properties. Surface of zeolites is very active with that, so they are fine catalysts for many processes. Size, form and properties of the surface of nano-size materials may be adapted and optimized for special application. Placing different ions in the pores of natural and synthetic zeolites we obtain modified materials, which have unique properties. Modification of zeolites is attained by the method of substitution of some ions for others in the channels of zeolites. Sorption properties of modified zeolites appear also at adsorption or substitution of ions in channels. Calculations of rate constant of the ionexchange process in the channels of the porous materials, during which one adsorbed particle is replaced by another, are presented below.

It is shown that for exchange process essential value has liquid reorganization in the channel of zeolite, distribution of electrostatic charges inside channel, interaction potential of adsorbed particle with channel wall. Calculation results may be used at zeolite's modification for different tasks.

\section{THEORETICAL CALCULATIONS}

Ion-exchange processes in the channels of zeolites (amorphous solid) considerably depend on geometric factors. For determinancy consider model, where steric restrictions for ionexchange processes are determined by channel's configuration. It will be assumed also, that the process passes by non-adiabatic mechanism.

Adsorbed particle will be considered as ion in polyatomic molecule. It is assumed that adsorbed particle is characterized by valent and deformative vibrations as ion in polyatomic molecule. The rest of the zeolite is considered as part of medium, which takes part in ion-exchange process. The second part of medium is liquid in the channel. For simplicity medium will be described in classical approximation. The following assumptions will be used for characterization of adsorbed particle: Valent and deformation vibration (for simplicity and clearness of results' statement) is considered as classical and harmonic.

Probability of ion-exchange process in classical approximation ${ }^{1-4}$ can be expressed as:

$$
\begin{aligned}
& \mathrm{W}_{\mathrm{fi}}=(\beta / \mathrm{i}) \Phi\left(\mathrm{R}^{*}, \varphi^{*}\right) \mathrm{L}_{\mathrm{fi}}^{2} \exp (-\beta \Delta \mathrm{F}) \int \mathrm{d} \theta \exp \left\{-\beta(1-\theta) \mathrm{U}_{\mathrm{i}}(\mathrm{R}, \varphi)\right. \\
& \left.-\beta \theta \mathrm{U}_{\mathrm{f}}(\mathrm{R}, \varphi)-\Psi^{\mathrm{m}}(\mathrm{R}, \varphi)\right\}^{*} \exp \left(-\beta \mathrm{T}_{\mathrm{e}}\right) * \delta\left(\mathrm{R}-\mathrm{R}^{\prime}\right) \delta\left(\varphi-\varphi^{\prime}\right)
\end{aligned}
$$

here $T_{e}$ is operator of kinetic energy of the particle; $R$ and $\varphi$ are the coordinates of the particles' atoms participating in the process and angles of their orientation in space; $\beta=\mathrm{kT}$, where $\mathrm{T}$ is temperature; $\mathrm{k}$ is Boltsmann constant; $\mathrm{U}_{\mathrm{i}}$ and $\mathrm{U}_{\mathrm{f}}$ are potential energies of the particles.

Particle on which is adsorbed particle will be denoted as 1; adsorbed particle will be denoted as 2; particle, which must substitute particle 2 will be denoted as 3 . It will be assumed, 
for simplicity, that both deformation vibrations of particles in molecules 1-2 and 1-3 are equal.

Potential energy of the molecule 1-2 in initial state can be expressed as:

$$
\mathrm{U}_{\mathrm{i}}=\mathrm{I}_{\mathrm{i}}+\mathrm{U}_{2}^{\delta}\left(\vartheta_{2}\right)+\mathrm{U}_{2}^{\mathrm{v}}\left(\mathrm{R}_{2}\right)+\mathrm{U}_{\mathrm{i}}^{\mathrm{s}}\left(\mathrm{R}_{2}, \mathrm{R}_{3}\right)
$$

Analogously in final state for molecule 1-3:

$$
\mathrm{U}_{\mathrm{f}}=\mathrm{I}_{\mathrm{f}}+\mathrm{U}_{3}^{\delta}\left(\vartheta_{3}\right)+\mathrm{U}_{3}^{\mathrm{v}}\left(\mathrm{R}_{3}\right)+\mathrm{U}_{\mathrm{f}}{ }^{\mathrm{s}}\left(\mathrm{R}_{2}, \mathrm{R}_{3}\right)
$$

here $\mathrm{I}_{\mathrm{i}, \mathrm{f}}$ coincides with dissociation energy of the molecule 1-2 and 1-3 in medium up to a sign, correspondingly indices $\mathrm{v}$ and $\delta$ are related to valent and deformation vibrations, index $\mathrm{s}$ is related to short-range interaction of particles 1-2 with 3 and 1-3 with 2 .

In Condon approximation for transfer probability we'll obtain $\left.^{1-4}\right]$ :

$$
\mathrm{W}_{\mathrm{fi}}=\frac{2 \pi \mathrm{R}_{2}^{2} \mathrm{R}_{3}^{2} \mathrm{~L}^{2}}{\mathrm{i} \mathrm{V}_{0}^{2}\left((1-\theta) \theta \mathrm{k}_{2}^{\mathrm{v}} \mathrm{K}_{3}^{\mathrm{v}}\right)^{1 / 2}} \exp (-\beta \theta \Delta \mathrm{F}-\beta \theta \Delta \mathrm{I}) \int_{0}^{\pi} \mathrm{d} \vartheta_{2} \sin \vartheta_{2} \int_{0}^{2 \pi} \mathrm{d} \varphi_{2}
$$

$\int_{0}^{\pi} \mathrm{d} \varphi_{3} \sin \vartheta_{3} \int_{0}^{2 \pi} \mathrm{d} \varphi_{3} \exp \left(-\beta(1-\theta)\left(\mathrm{U}_{2}^{\delta}+\mathrm{U}_{\mathrm{i}}^{\mathrm{s}}\left(\vartheta_{2}^{\prime}\right)\right)-\beta \theta\left(\mathrm{U}_{3}^{\delta}+\mathrm{U}_{\mathrm{f}}^{\mathrm{s}}\left(\vartheta_{3}^{\prime}\right)\right)\right)$ here $\mathrm{k}_{2}{ }_{2}$ and $\mathrm{k}_{3}^{\mathrm{v}}$ are force constants of valent vibrations of molecules $1-2$ and 1-3, $\vartheta_{3}^{\prime}$ is angle between vectors $\vec{R}_{3}$ and $\vec{R}_{2}$.

Exponentially-increased function at distance equal to sum of radiuses of particle $r_{0}$ can be taken as short-range potential.

As a result, for probability of transfer we'll obtain:

$$
\begin{aligned}
& \mathrm{W}_{\mathrm{fi}}=\frac{(2 \pi)^{3 / 2} \mathrm{R}_{2}^{2} \mathrm{R}_{3}^{2} \mathrm{~L}^{2} \sin ^{1 / 2}\left(\vartheta_{0}-\vartheta_{2}\right)}{\mathrm{i} \mathrm{V}_{0}^{2} \beta \theta\left(\beta \theta\left((1-\theta) \theta \mathrm{k}_{2}^{\mathrm{v}} \mathrm{K}_{3}^{\mathrm{v}}\right)^{1 / 2}\left(\mathrm{U}_{3}^{\prime}\right)^{3 / 2} \sin ^{1 / 2} \vartheta_{0} \sin ^{1 / 2} \vartheta_{2}\right.} \\
& \exp (-\beta \theta \Delta \mathrm{F}-\beta \theta \Delta \mathrm{I}) \int_{0}^{2 \pi} \mathrm{d} \varphi_{2} \int_{0}^{\pi} \mathrm{d} \vartheta_{2} \sin \vartheta_{2} \exp \left(-\beta(1-\theta) \mathrm{U}_{2}^{\delta}-\beta \theta \mathrm{v}_{\mathrm{f}}\right)
\end{aligned}
$$

$\exp \left(-\beta \theta \mathrm{U}_{3}^{\vartheta^{\prime}}\left(\vartheta_{0}-\vartheta_{2}\right)\right)$

where $\vartheta_{0}$ is significance of the angle at maximal approach of particles $\mathrm{v}_{\mathrm{i}}=\mathrm{U}_{\mathrm{i}}^{\mathrm{s}}\left(\vartheta_{3}=\vartheta_{0}\right)$.

Residual integration is conducted by $\varphi_{2}$ precisely and by $\vartheta_{2}$ with the help of inflection method.

For rate constant of the process it is obtained:

$$
\begin{aligned}
& \mathrm{K}_{\mathrm{n}}=\frac{|\beta \mathrm{L}|^{2} \mathrm{~V}_{\mathrm{r}}^{2} \beta \mathrm{k}_{2}^{\delta}\left(\beta \mathrm{k}_{2}^{\mathrm{v}}\right)^{1 / 2}}{2 \pi \beta \mathrm{R}_{20}^{2}\left(\beta \mathrm{E}_{\mathrm{r}}^{\mathrm{m}}+\left(2 \theta^{*}\right)^{-2} \mathrm{U}_{2}^{\vartheta} \partial \vartheta_{2}^{*} / \partial \theta\right)^{1 / 2}} \\
& \exp \left(-\beta \Delta \mathrm{F}-\beta\left(\mathrm{v}_{\mathrm{i}}+\mathrm{U}_{2}^{\delta}\left(\vartheta_{2}^{*}\right)+\left(\theta^{*}\right)^{2} \mathrm{E}_{\mathrm{r}}^{\mathrm{m}}\right)\right)
\end{aligned}
$$

where $V_{r}$ is reactionary volume and $\theta^{*}$ is defined from equation:

$$
\mathrm{U}_{2}^{\delta}-\mathrm{U}_{3}^{\delta}-(1-2 \theta) \mathrm{E}_{\mathrm{r}}^{\mathrm{m}}-\Delta \mathrm{F}-\mathrm{v}_{\mathrm{f}}+\mathrm{v}_{\mathrm{i}}=0
$$

It must be mentioned, that harmonic approximation for deformation vibrations was used nowhere. Besides, proposed method of rate constant calculation of ion-exchange process allows to consider short-range forces in different (non-exponential) form.

Obtained results may be used for rate constant evaluation of the process of substitution of one type ions for another in the channels of zeolites.

\section{Conclusion}

Processes of nonadiabatic ion-exchange in the channels of zeolites have been considered. Calculations of rate constant of ion-exchange process in the channels of zeolites, where one adsorbed particle is substituted for another, have been carried out. Analytical expressions for characteristic parameters of the process have been obtained. It is shown, that for exchange process essential value has liquid reorganization in the channel of zeolite, distribution of electrostatic charges inside channel, interaction potential of adsorbed particle with channel wall. Calculation results may be used at zeolite's modification for different tasks.

\section{REFERENCES}

1. A.A. Abrikosov, L.P. Gorkov and L.E. Dzyaloshonski, Methods of Quantum Field Theory in Statistical Physics, Dover Publications Inc., New York (1975).

2. R.R. Dogonadze and T.A. Marsagishvili, in ed.: R. Dogonadze, The Chemical Physics of Solvation, Part A, Elsevier Publ. Co., Amsterdam, p. 39 (1985).

3. P.M. Platzman and P.A. Wolff, Waves and Interactions in Solid State Plasmas, Academic Press, N.Y., London (1973).

4. T.A. Marsagishvili, J. Electroanal. Chem., 450, 47 (1998) 